\title{
A sequential Monte Carlo framework for noise filtering in InSAR time series
}

\author{
Mehdi Khaki, Mick S. Filmer, W. E. Featherstone, Michael Kuhn, Luyen K. Bui, Amy L. Parker
}

\begin{abstract}
This study proposes an alternative filtering technique to improve interferometric synthetic aperture radar (InSAR) time series by reducing residual noise while retaining the ground deformation signal. To this end, for the first time, a data-driven approach is introduced, which is based on Takens's method within the sequential Monte Carlo framework, allowing for a model-free approach to filter noisy data. Both Kalmanbased and particle filters are applied within this framework to investigate their impact on retrieving the signals. More specifically, particle filter (PF) and particle smoother (PaSm; to avoid confusion with persistent scatterers PS) are tested for their ability to deal with non-Gaussian noise. A synthetic test based on simulated InSAR time series, as well as a real test, are designed to investigate the capability of the proposed approach compared with spatio-temporal filtering of InSAR time series. Results indicate that PFs and more specifically $\mathrm{PaSm}$ perform better than other applied methods, as indicated by reduced errors in both tests. Two other variants of PF and adaptive unscented Kalman filter (AUKF) are presented and are found to be able to perform similarly to $\mathrm{PaSm}$ but with reduced computation time. The study suggests that PFs tested here could be applied in InSAR processing chains.
\end{abstract}

Index Terms-InSAR, non-Gaussian noise, data-driven technique, particle filter, sequential technique

\section{INTRODUCTION}

$\mathbf{M}$ ONITORING deformation (primarily subsidence/uplift) of the Earth's surface is important to understand its physical processes and the resulting hazards, e.g. earthquake, volcanoes, landslides, anthropogenic subsidence or uplift. Various techniques are employed to do this, but interferometric synthetic aperture radar (InSAR) has become a standard tool to undertake such studies by remotely sensing large areas at high spatial and temporal resolutions (e.g., [1]). In principle, InSAR uses two complex SAR images from repeat satellite passes to produce an interferogram over the common area. For a review of InSAR fundamentals see, e.g., [2].

Individual interferograms are highly sensitive to various noise sources such as those attributed to spatial and temporal decorrelation [3], atmospheric (e.g., [2]), topographic effects, thermal noise and orbit errors (e.g., [4]). These can affect the sought-after deformation signal, both in the estimated velocities and the time-series. Advances in processing methods such as multi-temporal InSAR where multiple interferograms

M. Khaki: School of Engineering, University of Newcastle, Callaghan, New South Wales, Australia. e-mail: Mehdi.Khaki@Newcastle.edu.au, Tel: $+61(2) 49216626$

M.S. Filmer, W.E. Featherstone, M. Kuhn, L.K. Bui, and A.L. Parker are with School of Earth and Planetary Sciences, Spatial Sciences, Curtin University, Perth, Australia. are 'stacked' (e.g., [5]), persistent scatterers (PS; e.g., [6]), small baseline subset (SBAS; [7]) and a combination of these approaches [8] can help reduce the effects of noise on the computed velocity, especially when a long time series with a large number of SAR scenes are available.

For deformation time series of interest to investigate geophysical events, the noise in the estimated displacement may be more problematic. The major source of noise in the InSAR time series is the combination of tropospheric delay, which comprise vertical stratification and turbulent components [2]. The vertical stratification component can be estimated by its relation with height (e.g., [9]), but the turbulent component is variable in flat areas, so more difficult to determine [10].

Various filtering methods are used at different stages of InSAR processing. For example, the Goldstein filter [11] is applied to the wrapped interferogram phase to reduce noise and improve the unwrapping process. A filter parameter $(\alpha)$ is implemented that can be set between zero and one; if set to zero, no filtering is done, while a setting of one will result in heavy filtering that is likely to significantly change the structure of the interferogram [12]. [8] uses a spectral filter on small baseline interferograms to assist in the selection of slowly decorrelating filtered phase pixels used in his combined SBAS and PS method. Spatio-temporal filtering (STF) is used by [13] in the Stanford Method for Persistent Scatters (StaMPS, see also [14]) on post-processed PS to reduce temporal noise in the time series (mostly time- and space-variable turbulent tropospheric noise, cf. [10]), and for spatial noise relative to other PSs. Like all filters, this can be set at different temporal and spatial scales, which will tend to remove noise, but may also remove parts of the sought-after deformation signal, particularly where the noise and deformation display similar characteristics in time and/or space [15].

Here, we focus on reducing residual noise, with a view to reducing processing and observation noise in the InSAR time series. A number of methods have been used to estimate this component (e.g., [16], [10]). The difficulty with applying such filters is that they can remove most noise present but at the cost of the geophysical signal of interest. Hence it is a balance to 'tune' the filter so that its spatial and temporal settings can be 'optimal' to remove as much noise as possible while retaining the information of interest. Notably, the spatiotemporal filtering employed in StaMPS assumes Gaussian noise, yet geophysical signals of interest may be non-linear in time, e.g., aseismic slip, or slow-moving landslides (see, e.g., [14]).

The main objective of this study is, therefore, to test 
some alternative filters that may improve upon results from spatio-temporal filtering (e.g., available in StaMPS) to reduce the noise in InSAR time series. Different studies have been undertaken to improve the signal to noise ratio in InSAR signals, especially prior to the unwrapping process (see, e.g., [11], [17]). These filters can generally be categorized into two groups, i.e., filtering in the spatial (e.g., [19], [20]) and frequency (e.g., [11], [21]) domains. These filters have been shown to be effective for smoothing as well as dealing with non-linear phase noises [22]. Nevertheless, there are a number of factors that degrade the performance of spatial and spectral filters. For example, multiple interferograms are required in most of these filters to better model noise, especially atmospheric noise [23]. The methods also rely excessively on coherent pixel-wised data, which may not always be available (e.g., [24]). The spatio-temporal filters, despite being easier to apply due to their simplicity, can alter or remove the fringe structures in the signals [22]. A number of studies have been put forward to address this (see, e.g., [25], [26]). Furthermore, the Gaussian noise assumption is a fundamental principle in a large group of filters, which in reality may not always be the case (e.g., [27]).

In order to address these problems, an alternative InSAR filtering approach is proposed here. The technique is based on a data-driven method, namely Takens's filter [28], [29], [30]. Our proposed approach implements the Takens method for attractor reconstruction within the sequential Monte Carlo framework, allowing for a model-free approach to filter noisy data (e.g., [31]). Based on Takens's theorem, equations underlying a model, which describe the time evolution of a system, e.g., land deformation time series, can be replaced by the information contained in the data. This, along with the implementation of Monte Carlo techniques such as Kalman filters (KFs) and particle filters (PFs) for updating the system based on the current data, allows the handling of noise in the observed data (e.g., [29]).

Here, for the first time, we apply the Takens filter to reduce residual noise in InSAR data. Contrary to previous studies that used KF and PF techniques for phase unwrapping and/or noise reduction (e.g., [32], [33]), the Takens method relies only on data and avoids assumptions on the state-space model noises and can be computationally faster (e.g., [30]). To assess the performance of the Takens method, we tested it on synthetic and real data and compared with [13] spatio-temporal filtering (STF) which is usually applied to post-processed PS.

\section{Methodology}

The proposed filtering scheme comprises two steps in a sequential process, i.e., forecast then analysis, similar to data assimilation techniques (e.g., [34], [35]). Sequential methods do not require an adjoint (like variational methods) and are becoming increasingly popular because of their reasonable computational requirements as they do not need to record and use historical data. In the forecast step, the state estimate at time $t$ (e.g., land deformation) and its corresponding probability density function (PDF) are forwarded in time to $t+1$ using a state transition operator. Monte Carlo methods are commonly used in the forecast step (based on ensembles or particles). These are then filtered in the analysis step based on the likelihood of the observations (e.g., simulated observations). Traditionally, a dynamical model is used in the forecast step, however, a problem arises when a model is not available. To address this, the Takens filter is applied to form the state transition operator (see Section II-A). Various filtering methods can then be used for the analysis step to update forecast PDF such as Kalman (e.g., ensemble Kalman filtering) or point-mass weight (e.g., PF). Here, three variants of most commonly used sequential techniques, i.e., adaptive unscented Kalman filter (AUKF), PF and particle smoother $(\mathrm{PaSm})$ are applied (see Sections II-B-II-C). We also test STF [13] to assess the capability of the proposed filtering method. The STF is 'tuned' to determine the 'optimal' settings for the comparison. This includes applying different values for various parameters used in the STF such as band-pass phase filters and low-pass cutoff to reach the best performance compared to the 'truth'. The corresponding outcomes are then used to evaluate the proposed filtering scheme.

\section{A. Takens's filter}

The Takens filter does not rely on a model and its corresponding equations, which significantly decreases the computational burden with comparable outcomes with respect to a standard case. The absence of a model in the InSAR time series filtering process makes the Takens filter a potential candidate to be used for forecasting. [29] showed that this filter has a high capability for time series filtering in the presence of various noises. The Takens method is used to reconstruct the model, e.g., following the terminology of [30] shown by $\mathbf{f}$, for the forecast step with a local proxy $\tilde{\mathbf{f}}$. This is done using a set of training data based on the InSAR time series to represent the state of the system. The training data is obtained from the delay vector as well as neighbouring grid points. The delay vector $\mathbf{x}$ (at $t$ ) can be formed using the historical state variables, e.g., $d$ temporal delays of InSAR line of sight (LoS), $\mathrm{x}^{\mathrm{o}}$, following,

$\mathbf{x}_{t}=\left[\mathbf{x}^{\mathbf{o}}{ }_{t}, \mathbf{x}_{t-1}^{\mathbf{o}}, \ldots, \mathbf{x}_{t-d}^{\mathbf{o}}\right]$.

Next, similar delay vectors located at the $N$ nearest neighbors, i.e., grid points located close to the point of interest (based on Euclidean distance) within a set of training data, are derived by,

$$
\begin{aligned}
& \mathbf{x}_{t}^{1}=\left[\mathbf{x}_{t}^{\mathbf{o}}, \mathbf{x}_{t-1}^{\mathbf{o}}, \ldots, \mathbf{x}_{t-d}^{\mathbf{o}}{ }_{t-d}\right], \\
& \mathbf{x}_{t}^{2}=\left[\mathbf{x}_{t}^{\mathbf{o}}, \mathbf{x}_{t-1}^{\mathbf{o}}, \ldots, \mathbf{x}_{t-d}^{\mathbf{o} 2}\right], \\
& \vdots \\
& \mathbf{x}_{t}^{N}=\left[\mathbf{x}_{t}^{\mathbf{o}}{ }_{t}, \mathbf{x}_{t-1}^{\mathbf{o} N}, \ldots, \mathbf{x}_{t-d}^{\mathbf{o} N}\right] .
\end{aligned}
$$

Once delay vectors are calculated, a local model can be created for the forecast step, i.e., to advance state from $t$ to $t+1$ and correspondingly $\mathbf{x}_{t}$ to $\mathbf{x}_{t+1}$. The local model $\tilde{\mathbf{f}}$ can then be generated (in its simplest from) using a weighted average of the training data according to

$\mathbf{x}_{t+1}=\omega_{1} \gamma^{1} \mathbf{x}_{t+1}^{1}+\omega_{2} \gamma^{2} \mathbf{x}_{t+1}^{2}+\ldots+\omega_{N} \gamma^{N} \mathbf{x}_{t+1}^{N}$, 
with

$\omega_{i}=\frac{\mathrm{e}^{-\left(d_{i} / \sigma\right)^{2}}}{\sum_{j=1}^{N} \mathrm{e}^{-\left(d_{j} / \sigma\right)^{2}}}$,

where the distance between the $j^{\text {th }}$ neighbour of $\mathbf{x}_{t}$ is indicated by $d_{i} . \sigma$ is applied to control the contribution of each neighbour in the local model and it is called a bandwidth parameter (here $\sigma=2$ ). $\gamma^{k}, \quad k=1, \cdots, N$ in Eq. (3) refers to the coherence factor, which is calculated as an absolute value of the correlation coefficient between two SAR scenes following

$\gamma_{1,2}=\frac{\left\|E\left(z_{1}, z^{*}{ }_{2}\right)\right\|}{\sqrt{E\left(\left\|z_{1}\right\|^{2}\right) \cdot E\left(\left\|z_{2}\right\|^{2}\right)}}$,

where $z_{1}$ and $z_{2}$ are the complex return for two SAR and $E($. represents the expectation function. The inclusion of coherence in the local model formulation decreases the effect of noisy observations with smaller $\gamma$. Afterwards, the forecast state $\mathbf{x}_{t+1}$ is updated in the next step based on Bayes's rule [34]. This is done to update the forecast state based on the current observations.

As shown by [29], the availability of unpredictable noise components in the observed data is a common context for the application of the Kalman-Takens filter, which is able to handle observation noise using sequential forecast and update steps. Applying this method to the entire training data set reduces the observation noise in the training data which will improve future filtering and also provide better neighbours to improve forecasting (see, [36]). The application of the Kalman-Takens filter also allows for better quantification of the uncertainty in the state, e.g., through the state forecast covariance matrix, which will then be reduced using the update step (see Sections II-B-II-C). While delay coordinate embedding replaces the missing model, the Kalman (or particle) update offers a maximum likelihood estimate of the reconstructed state in the presence of noise. This combination of two filters, therefore, contains complementary strengths of the two.

Three different methods from sequential Monte Carlo framework are used for the update step. These include AUKF ([37]), PF ([38]), and PaSm ([39]). The Kalman-based and PFs are specifically selected due to their ability to deal with different types of noise. Where non-Gaussian noise exists, the particle approach is particularly well suited (e.g., [38]). These filters are based on point mass representations of probability densities, which generalise the traditional Kalman filter and do not rely on a Gaussian noise assumption.

\section{B. $A U K F$}

Adaptive unscented Kalman filter (AUKF) is based on the Monte Carlo scheme, which generates random variables and propagates them through a non-linear function using a deterministic sampling approach for producing $2 L+1$ sigma points with $L$ being the dimension of the state as

$\mathbf{x}_{t}^{0}=\mathbf{x}_{t}$,

$\mathbf{x}_{t}^{i}=\mathbf{x}_{t}+\left(\sqrt{(L+\lambda) \mathbf{P}_{t}}\right)_{i} \quad i=1, \ldots, L$,

$\mathbf{x}_{t}^{i+L}=\mathbf{x}_{t}-\left(\sqrt{(L+\lambda) \mathbf{P}_{t}}\right)_{i} \quad i=1, \ldots, L$,

where $\mathbf{P}_{t}$ represents the state covariance matrix. The associated weights to the sigma points are estimated by

$w_{s}^{0}=\frac{\lambda}{(L+\lambda)}$,

$w_{c}^{0}=\frac{\lambda}{(L+\lambda)}+\left(1-\alpha^{2}+\beta\right)$,

$w_{s}^{i}=\frac{1}{2(L+\lambda)} \quad i=1, \ldots, 2 L$.

$\lambda$ is the scaling parameter with $\lambda=\alpha^{2}(L+\beta)-L$. $\alpha(0-1)$ controls the spread of the sigma points and $\beta$ is usually set to 0 ([40]). The generated sigma points are then integrated with a model using the local proxy $\tilde{\mathbf{f}}$, created in the KalmanTakens filter to estimate the forecast state. Once done, the forecast averages and corresponding covariance matrices are calculated following [40], by

$\begin{aligned} \mathbf{x}_{t+1}^{f} & =\sum_{j=0}^{2 L} \mathbf{w}_{s}^{j} \mathbf{x}_{t+1}^{f, j}, \\ \mathbf{y}_{t+1}^{f} & =\sum_{j=0}^{2 L} \mathbf{w}_{s}^{j} \mathbf{H} \mathbf{x}_{t+1}^{f, j},\end{aligned}$

where $\mathbf{H}$ is the observation operator that maps the model states into the observation space to update state estimates. Next, the analysis step is employed, which updates the forecast state $\mathbf{x}_{t+1}^{f}$ using incoming observations $\mathbf{y}_{t+1}$ to calculate the analysis state $\mathbf{x}_{t+1}^{a}$ based on the Kalman update equations,

$\mathbf{x}_{t+1}^{a}=\mathbf{x}_{t+1}^{f}+\mathbf{K}\left(\mathbf{y}_{t+1}-\mathbf{y}_{t+1}^{f}\right)$,

with $\mathbf{K}$ being the Kalmna gain and calculated according to covariance matrices associated with the process $\left(\mathbf{Q}_{t}\right)$ and observation ( $\mathbf{R}_{t+1}$ ) (see more details in [40]). Critical to the success of this method is the selection of the filter noise covariance, and in particular the process noise covariance matrix Q. [37] show that unscented Kalman filter performance is highly dependent on $\mathbf{Q}$, especially for non-linear problems. Here, we use the method of [37] to adaptively estimate this covariance matrix. The general idea of [37] is to use the increment, $\epsilon_{t}=\mathbf{y}_{t}-\mathbf{y}_{t}^{f}$, to estimate the noise covariance at each time step, as per [37].

\section{Particle filter}

Particle filter $(\mathrm{PF})$ is also a sequential Monte Carlo method, which represents the state PDF by a set of weighted particles [38]. The state PDF is then decomposed as

$P\left(\mathbf{x}_{t} \mid \mathbf{y}_{1: t}\right) \approx \sum_{i=1}^{M} \omega_{t}^{i} \delta\left(\mathbf{x}_{t}-\mathbf{x}_{t}^{i}\right)$,
3

4
5

6 
where $\left\{\mathbf{x}_{t}^{i} ; i=1 \ldots M\right\}$ (with $M$ being the particle size) are the particles at time $t$, observations between time 1 and $t$ are denoted by $\mathbf{y}_{1: t}, \omega_{t}^{i}$ are the weights of the particles (normalised importance weight), and $\delta$ is the Dirac delta function. In the forecast step, PF just integrates the particles forward with the local proxy $\tilde{\mathbf{f}}$, exactly as AUKF, and their weights remain the same. In the analysis step, only the weights, and not the particles, are updated using

$\omega_{t}^{i}=\frac{P\left(\mathbf{y}_{t} \mid \mathbf{x}_{t \mid t-1}^{i}\right)}{\sum_{j} P\left(\mathbf{y}_{t} \mid \mathbf{x}_{t \mid t-1}^{j}\right)}$.

PaSm is also applied in a largely similar manner by propagating particle members and their associated weights in the forecast step and updating the latter in the analysis step. The main difference between the two approaches is estimation of the state distribution. Contrary to PF, the distribution at a particular time is calculated in PaSm using all of the observations up to some later time. This results in using additional information (e.g., taking advantage of a few later observations), which leads to smoother estimates than PF and likely to a better performance, especially if state estimation at a particular time is not required instantly [39]. A forward-backward smoother is assumed here, which proceeds by making first a forward filtering pass to compute the filtered distribution at each time step, and then a backward smoothing pass to determine the smoothing distribution [41]. The distribution can be approximated by

$P\left(\mathbf{x}_{t} \mid \mathbf{y}_{1: T}\right) \approx \sum_{i=1}^{M} \omega_{t \mid T}^{i} \delta\left(\mathbf{x}_{t}-\mathbf{x}_{t}^{i}\right)$,

with $\mathbf{y}_{1: T}=\left\{\mathbf{y}_{1} \ldots \mathbf{y}_{T}\right\},(T \geq t+1)$ and corresponding particle weights of

$\omega_{t \mid T}^{i}=\omega_{t}^{i}\left[\sum_{j=1}^{M} \omega_{t+1 \mid T}^{j} \frac{P\left(\mathbf{x}_{t+1}^{j} \mid \mathbf{x}_{t}^{i}\right)}{\sum_{k=1}^{N} \omega_{t}^{k} P\left(\mathbf{x}_{t+1}^{j} \mid \mathbf{x}_{t}^{k}\right)}\right]$.

A resampling technique of systematic resampling ([42]) is applied to account for the so-called 'degeneracy problem' in which the weights of all particles become negligible except only for a very few, requiring a prohibitive number of particles to prevent the particles collapsing [38]. The method draws only one random number $u_{1} \sim U(0,1 / N)$ and the remaining $N-1$ numbers are then calculated from $u_{1}$ as

$U_{i}=u_{1}+\frac{(i-1)}{M}, \quad i=2 \ldots M$.

These are then used to select a new set of particles according to the multinomial distribution [38]. Figure 1 outlines a summary of the filtering framework and the applied filters for the InSAR noise reduction process in this study.

\section{EXPERIMENT SETUPS}

\section{A. Synthetic data}

InSAR line of sight (LoS) phase observations and their associated errors are simulated following [23] and [18] with similar average properties to the TerraSar-X satellite mission [43]. The simulation is done over the Perth (Western Australia)

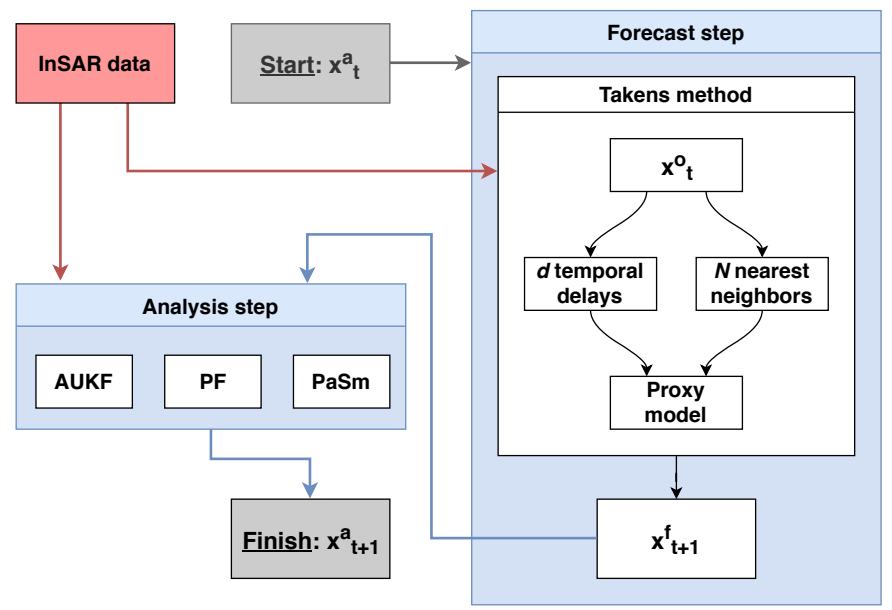

Fig. 1: A schematic illustration of the filtering steps based on the Takens method, as well as sequential techniques, i.e., AUKF, PF, and PaSm at every assimilation cycle.

metropolitan region with 11-day repeats from October 2012 to October 2016 within the spatial extents $32.25^{\circ} \mathrm{S}$ to $31.65^{\circ} \mathrm{S}$ and $115.60^{\circ} \mathrm{E}$ to $116.10^{\circ} \mathrm{E}$ with $\sim 30 \mathrm{~m}$ pixel size. Details of the steps taken to generate synthetic data are described in the supplementary materials. In summary, the process includes introducing land subsidence using the point pressure model, simulating topographic errors, atmospheric artefacts, orbital errors, and temporal decorrelations as per [18], and lastly creating interferograms. We then apply the 3D phase unwrapping method in StaMPS small baseline MTI [14]. Once the synthetic interferograms are produced, they are merged and converted to deformation time-series. To this end, small baseline subset (SBAS) is used with the spatial perpendicular baselines less than $150 \mathrm{~m}$ and temporal baselines less than 100 days. The proposed filtering methods are then applied to the simulated LoS data. These synthetic data are simpler than real interferograms and do not include, e.g., nonlinear phasetopography gradients and quadratic orbital ramps. Nevertheless, they can sufficiently reproduce the primary features of InSAR data for testing the proposed filters. For an additional analysis, real data will be considered in Section III-B.

\section{B. Real data}

The InSAR component of the real test data used $109 \mathrm{X}$ band scenes (wavelength $3.1 \mathrm{~cm}$ ) over Perth (Western Australia) from the TerraSAR-X (TSX) satellite mission under the German Aerospace Centre (DLR) science project LAN1499. The scenes were acquired between October 2012 and October 2016, with a temporal resolution of 11 days but with some gaps due to operational priorities for the satellite. The 109 scenes were used to process 442 SBAS interferograms using the Doris software [44] within StaMPS small baseline MTI processing [14]. The slowly decorrelating filtered phase (SDFP) pixels [8], were down-sampled to $30 \mathrm{~m}$ spatial resolution using the procedure described in [14] to reduce the many millions of SDFP pixels to a manageable dataset. Long-wavelength orbit and ionospheric effects were accounted for via the estimation
1 
and removal of a phase ramp, which is suitable for the TSX scene extent of $30 \times 50 \mathrm{~km}$ [14]. The LoS deformation time series was computed from the SBAS interferogram SDFP differences.

The time series is filtered to reduce the residual noise using various filters including the proposed Takens approach. The results are then compared with GPS time series over the study period (October 2012 to October 2016), which is provided by Nevada Geodetic Laboratory (http://geodesy.unr.edu/) for the international GNSS service (IGS) station "PERT" in Perth GPS data are used to assess the performance of the InSAR filters in the real test, albeit for a single site and not whole area.

\section{RESUlTS AND DisCUSSIONS}

\section{A. Synthetic test}

An evaluation of the proposed filters, also compared to the existing method of STF, is assumed in this section. The main objective is to reduce the impact of noise by applying different filters and retrieve the artificially introduced subsidence signals from the simulated phase. To this end, all the filters are applied to the synthetic data using their 'optimum' configurations and their results are validated against the unperturbed data (the simulated "truth"). Figure 2 depicts the time series (at a single location) from the perturbed (red) and unperturbed (green) LoS deformation, as well as the results of each filter applied (blue). More time series comparisons over different points can also be found in the supplementary materials. Moreover, detailed results can be found in Table I, where maximum differences, RMSE, standard deviation (STD) and correlation coefficients are reported. In Table I, average results over the entire scene are presented. Figure 2 shows that the application of all the filters decreases the misfits with respect to the unperturbed displacements. The level of improvement, however, is different for different filters, as it can also be seen from the indicated average error for each case. These errors are calculated as the average of absolute differences between the filtered time series and the unperturbed time series. The particle filters, and particularly PaSm, achieve the 'best' results. We gauge 'best' as the closest agreement with the simulated "truth" as determined by the lowest RMSE and highest correlation coefficient (cf. Table I). PaSm reduces RMSE from $10.30 \mathrm{~mm}$ in the perturbed data to $6.63 \mathrm{~mm}$ (a reduction of 36\%).

The weakest performance belongs to the STF based on both Figure 2 and Table I. The correlation between filtered and unperturbed ("truth") time series for the STF is 0.57 , smaller than PF (0.78) and PaSm (0.89). From Figure 2, in general, smoother time series are obtained from the AUKF, which is observed to better alleviate high-frequency noise than STF and PF. This resulted in smaller STD $(6.17 \mathrm{~mm})$ compared to STF $(7.34 \mathrm{~mm})$ and PF $(6.81 \mathrm{~mm})$. PaSm is found to better improve the time series, which contrary to other filters, do not rely on a Gaussian noise assumption. This allows the particle approaches to model the errors more realistically, leading to better performance especially for particle smoother, e.g., with the lowest RMSE $(4.22 \mathrm{~mm})$ and highest correlation (0.89). The most promising results among the three proposed filter variants also belong to $\mathrm{PaSm}$, slightly better than $\mathrm{PF}$, which can be attributed to the applied forward-backward algorithm allowing for using more information in the filtering process.

Spatial distributions of the displacement errors (i.e., the misfit between the results and the simulated "truth") based on the simulated data are demonstrated in Figure 3. At each grid point, the temporally averaged error (i.e. average of error time series at each grid point) is calculated for all these filters, as well as when no filter (noisy data) is applied. Smaller errors are found from PF and PS, and to a lesser degree AUKF, than STF. These can be inferred from the lower differences over the entire area, which supports the previous results indicating the superiority of the proposed methods. Another major discrepancy among the STF errors maps with those of new filters refers to a smoother spatial pattern in the latter (cf. Figure 3). The smoother errors maps of PF, PaSm and AUKF can be explained by the use of neighboring points in reconstructing the model for the Takens approach. The proxy model is constructed from $N$ neighbour points, which means implicit spatial smoothing is applied in the filter's forecast steps. Figure 3 also shows that the PaSm results in a smoother spatial pattern and smoother error time series than PF due to the former implementing the filtering followed by a smoothing process (e.g., [45]), while the latter only applies filtering.

Larger errors can be found around the imposed deformation source. Such larger error amplitudes are more pronounced in the STF and AUKF 'error' maps, yet, much less than the error map of noisy data. To better represent this, the filters' RMSE results at constant distances from the deformation source are compared in Figure 4. For this purpose, RMSE values are spatially interpolated to these radial distances using the nearest neighbour approach. As the distance increases, the error values for all filters decrease but this is more noticeable for STF, which demonstrates the largest error close to the deformation source. Again, PaSm and to a lesser degree PF hold the best performance slightly better than AUKF and considerably better than STF. Overall, similar to previous results, the particle approaches (PF and $\mathrm{PaSm}$ ) obtain 'better' results against the other applied filters.

\section{B. Real test}

To further investigate the performance of the filters in a more realistic scenario, their results from real InSAR data from TerraSar-X are compared with GPS time series. This is not an optimal evaluation as GPS data are subject to various error sources, which can be different than those in InSAR data. However, in the absence of reliable validation data, such an evaluation may provide some insight into which filtering method can better reflect the land displacements albeit at one point. Figure 5 displays time series of InSAR data (red), GPS (green), and filtered time series (blue). The comparison is done over a GPS station between InSAR data filtered by various methods and GPS time series (cf. Figure 5), which is converted to LoS data based on the TerraSar-X satellite look and heading angles (following Eq. (1) in [46]). The correlation values, i.e., between each two time series are also indicated for each case. Improvements can be seen for all the applied filters based on 

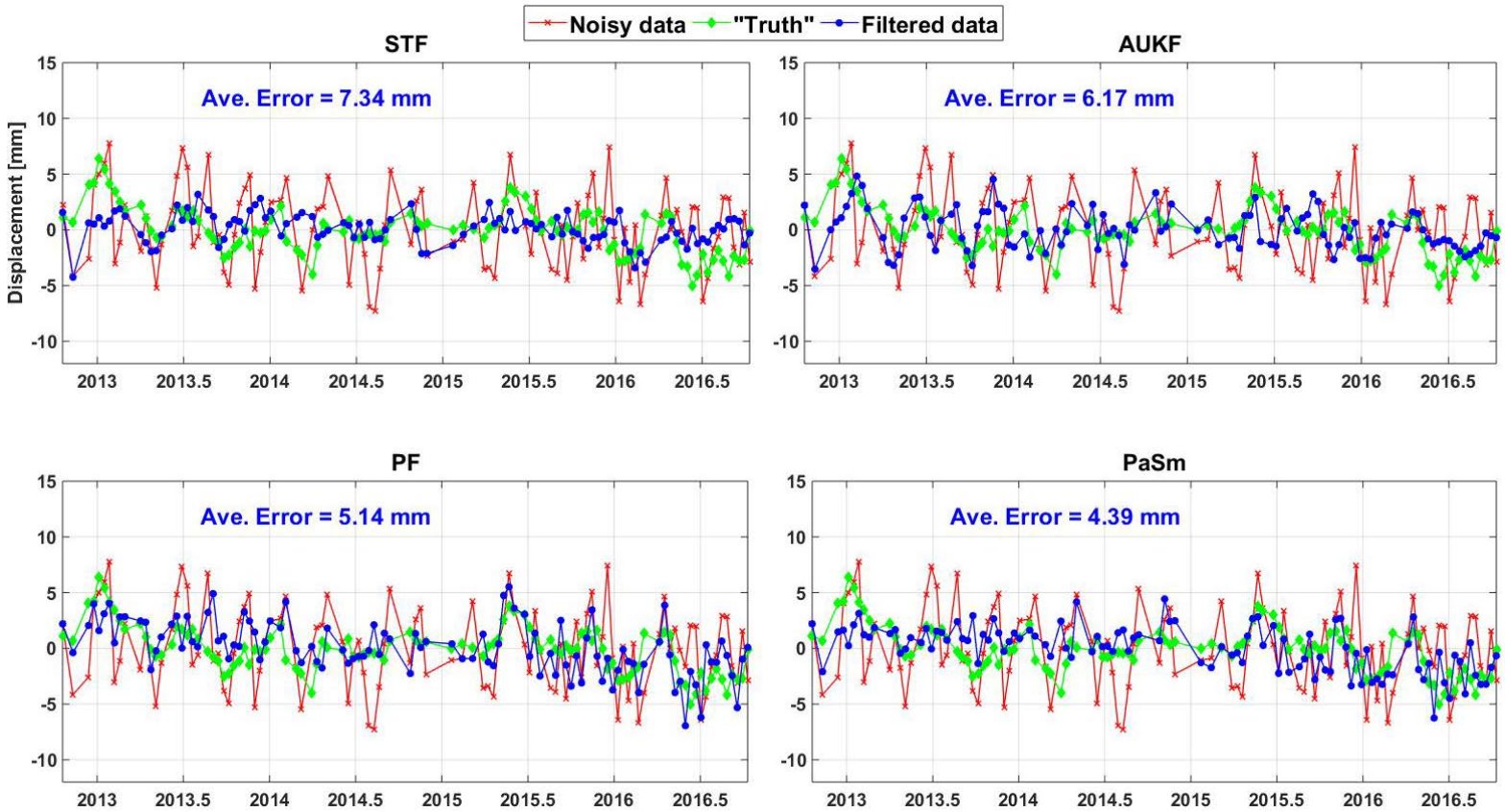

Fig. 2: InSAR time series at a randomly selected point (latitude $-31.802^{\circ}$ and longitude $115.885^{\circ}$ ) from different filtering scenarios based on the synthetic data compared to the no filter results and the "truth". Note that average errors between the filtered times series and "truth" is indicated on each subfigure. The average error of noisy (unfiltered) data is $10.29 \mathrm{~mm}$.

TABLE I: Summary of statistics derived from implementing different filters (with respect to 'Truth') on the simulated InSAR time series (2012-2016 for all filters) and over the whole area. RMSE reduction is with respect to original data (i.e. no filter applied).

\begin{tabular}{lccccc}
\hline Filter & Max error $(\mathrm{mm})$ & RMSE $(\mathrm{mm})$ & STD $(\mathrm{mm})$ & Correlation & RMSE reduction (\%) \\
\hline No filter & 13.35 & 10.30 & 9.72 & 0.38 & - \\
STF & 12.08 & 8.56 & 7.34 & 0.57 & 17 \\
AUKF & 9.78 & 7.46 & 6.81 & 0.66 & 28 \\
PF & 9.35 & 7.11 & 6.17 & 0.78 & 31 \\
PaSm & 7.49 & 6.63 & 4.22 & 0.89 & 36 \\
\hline
\end{tabular}

the better agreement between the filtered results and GPS data. Nevertheless, better results are provided by particle filters. In some time periods, e.g., after 2015, large misfits between the STF results and GPS observations exist. AUKF, PF and PaSm, on the other hand, provide better results (smaller differences to GPS), which shows their ability to better capture changes. However, larger correlation values are obtained between PF and PaSm results and GPS data. Overall, AUKF, PF, and PaSm time series indicate better agreement with the GPS data. The best performance is obtained from applying PaSm with 0.74 correlation to GPS time series against 0.34 for STF, 0.53 for AUKF, and 0.69 for PF. These results agree with the synthetic experiment results, in which particle filters also performed better.

\section{CONCLUSION}

An alternative InSAR noise filtering scheme was proposed in this study, which comprises different steps including the Takens method for attractor reconstruction, followed by the sequential Monte Carlo framework, allowing for a modelfree approach to filter noisy data. The method has significant benefits because of its capability to deal with non-Gaussian noise. A synthetic test was designed based on the simulated InSAR LoS phase data to investigate the capability of the proposed approach compared with the spatio-temporal filtering with the results indicating that the particle approaches (PF and $\mathrm{PaSm}$ ), and to a lesser degree AUKF, show a larger reduction in residual noise, both temporally and spatially. Specifically, PaSm successfully reduced RMSE by $34 \%$, approximately $5 \%$, $10 \%$, and $15 \%$ better than PF, AUKF, and STF, respectively. Moreover, Particle filters, especially $\mathrm{PaSm}$ appear to agree better with GPS in a real test. The capability of PaSm and 3 4 5 6 7 8 


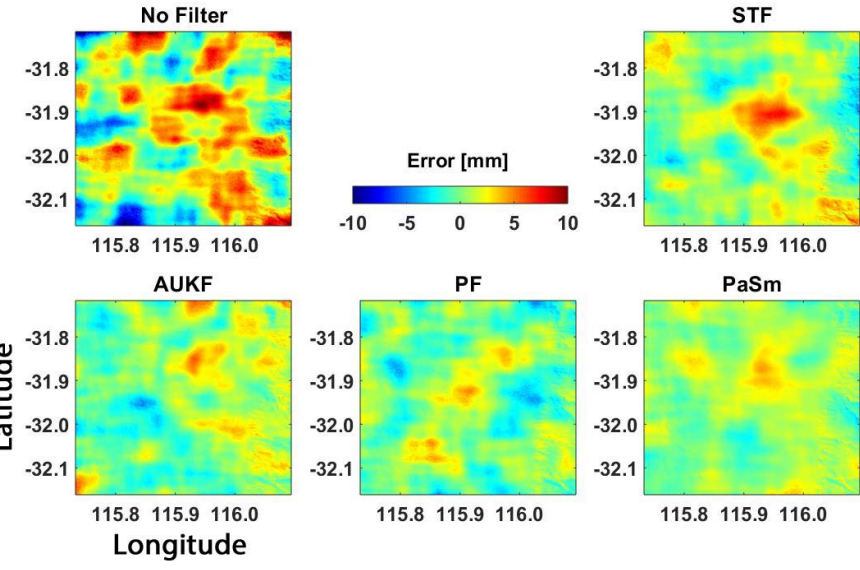

Fig. 3: Temporally averaged 'error' maps from different filtering scenarios $(\mathrm{mm})$. Error is the difference between the filtered results and "truth" data.

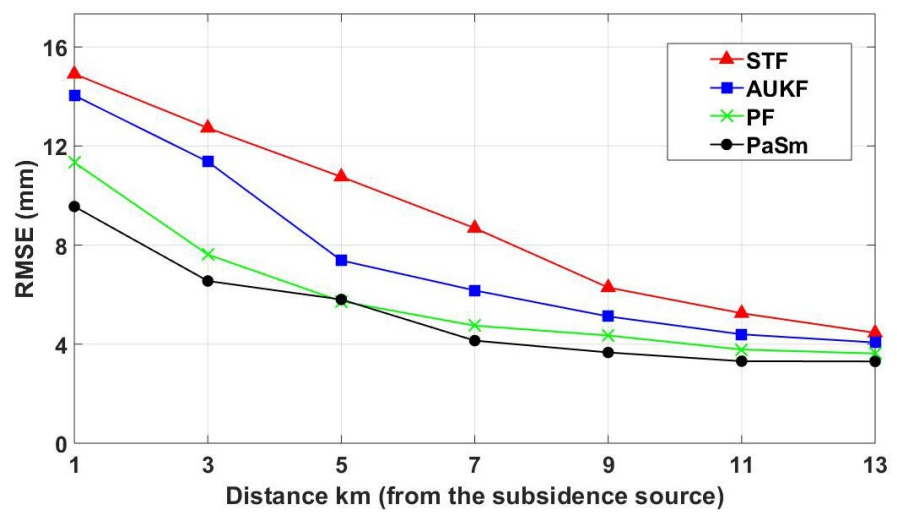

Fig. 4: The filters' average RMSE results at constant distances from the simulated deformation source.
PF in dealing with non-Gaussian noise can explain their better performance, especially against the Kalman-based filter AUKF, however, for the PaSm, this was obtained at the expense of a heavier computational burden. PF, on the other hand, achieved the closest performance amongst other filters in a more efficient manner, e.g., in terms of processing time and complexity. The next alternative can be AUKF, which is highly capable of reducing noise using much less population size. These results suggest that the particle filters tested here using the Takens approach can offer alterative methods for dealing with noisy InSAR signals, which depends on the filters' optimization.

\section{ACKNOWLEDGMENT}

The authors would like to thank German Aerospace Centre (DLR) science project LAN1499 for providing TSX data, and Nevada Geodetic Lab for providing GPS data. We also acknowledge A. Hooper for StaMPS software.

\section{REFERENCES}

[1] Massonnet, D., Rabaute, T. (1993), Radar interferometry: Limits and potential, IEEE Trans. Geosci. Remote Sens., 31,455- 464.

[2] Hanssen, R.F. (2001), Radar Interferometry: Data Interpretation and Error Analysis, 308 pp., Springer, New York.
[3] Zebker, H.A., Villasenor, J. (1992), Decorrelation in interferometric radar echoes, IEEE Transactions on Geoscience and Remote Sensing, 30(5), 950-959, doi: 10.1109/36.175330.

[4] Bahr, H., Hanssen, R.F. (2012), Reliable estimation of orbit errors in spaceborne SAR interferometry: The network approach, Journal of Geodesy, 86(12), 1147-1164, doi: 10.1007/s00190-012-0571-6.

[5] Sandwell, D.T., Price, E.J. (1998), Phase gradient approach to stacking interferograms, J. Geophys. Res., 103( B12), 30183- 30204, doi: 10.1029/1998JB900008.

[6] Ferretti, A.,Prati, C., FRocc, a. (2001), Permanent scatterers in SAR interferometry IEEE Trans. Geosci. Remote Sens., 39 (1), 8-20.

[7] Berardino, P., Fornaro, G., Lanari, R., Sansosti, E. (2002), A new algorithm for surface deformation monitoring based on small baseline differential SAR interferograms, IEEE Transactions on Geoscience and Remote Sensing, 40, 2375-2383.

[8] Hooper, A. (2008), A multi-temporal InSAR method incorporating both persistent scatterer and small baseline approaches, Geophysical Research Letters, 35(16), L16302, doi: 10.1029/2008GL034654.

[9] Bekaert, D., Hooper, A., Wright, T. (2015), Reassessing the 2006 Guerrero slow slip event, Mexico: implications for large earthquakes in the Guerrero Gap, Journal of Geophysical Research, 120, doi: 10.1002/2014JB011557.

[10] Cao, Y., Li ,Z., Wei, J., Hu, J., Duan, M., Feng, G. (2018), Stochastic modeling for time series InSAR: emphasis on atmospheric effects, Journal of Geodesy, 92(2):185-204, doi: 10.1007/s00190-017-1055-5.

[11] Goldstein, R.M., Werner, C.L. (1998), Radar interferogram filtering for geophysical applications, Geophysical Research Letters, 25(21): 40354038, doi: 10.1029/1998GL900033.

[12] Baran, I., Stewart, M.P., Kampes, B.M., Perski, Z. (2003), Lilly, P. A modification to the goldstein radar interferogram filter. IEEE Trans. Geosci. Remote Sens. 2003, 41, 2114-2118.

[13] Hooper, A., Segall, P., Zebker, H. (2007), Persistent scatterer interferometric synthetic aperture radar for crustal deformation analysis, with application to Volcan Alcedo, Galapagos, Journal of Geophysical Research: Solid Earth, 112(B7), B07407, doi:10.1029/2006JB004763.

[14] Hooper, A., Bekaert, D., Spaans, K., Arikan, M. (2012), Recent advances in SAR interferometry time series analysis for measuring crustal deformation. Tectonophysics, 514-517: 1-13, doi: 10.1016/j.tecto.2011.10.013.

[15] Sun, Q., Li, Z., Zhu, J., Ding, X.1., Hu, J., Xu, B. (2013), Improved Goldstein filter for InSAR noise reduction based on local SNR, Journal of Central South University, 20: 1896, doi: 10.1007/s11771-013-1688-3.

[16] Fattahi, H., Amelung, F. (2015), InSAR bias and uncertainty due to the systematic and stochastic tropospheric delay, Journal of Geophysical Research: Solid Earth, 120(12), 8758-8773, doi: 10.1002/2015JB012419.

[17] Schmidt, D., Burgmann, R. (2003), Time-dependent land uplift and subsidence in the Santa Clara valley, California, from a large interferometric synthetic aperture radar data set. Journal of Geophysical Research Solid Earth, 108(B9), 2416, doi: 10.1029/2002JB002267.

[18] Lee, C.W., Lu, Z., Jung, H.S. (2012), Simulation of time-series surface deformation to validate a multi-interferogram InSAR processing technique, International Journal of Remote Sensing, 33(22), 7075-7087, doi: 10.1080/01431161.2012.700137.

[19] Candeias, A.L.B., Mura, J.C., Dutra, L.V., Moreira, J.R. (1995), Interferogram phase noise reduction using morphological and modified median filters, Proceedings IGARSS95 1: 166-168, doi: 10.1109/IGARSS.1995.519679.

[20] Lanari, R., Fornaro, G., Riccio, D., Migliaccio, M., Papathanassiou, K.P., Moreira, J.R., Schwabisch, M., et al. (1996), Generation of digital elevation models by using Sir-C/X-SAR multifrequency two-pass interferometry: The Etna case study, IEEE Transactions on Geoscience and Remote Sensing, 34(5), 1097-1114, doi: 10.1109/36.536526.

[21] Huang, N.E., Shen, Z., Long, S.R., Wu, M.C., Shih, H.H., Zheng, Q., Yen, N.C., Tung, C.C., Liu, H.H. (1998), The empirical mode decomposition and the Hilbert spectrum for nonlinear and non-stationary time series analysis, Proceedings of the Royal Society of London, Series A: Mathematical, Physical and Engineering Sciences, 454: 903-995, doi: 10.1098/rspa.1998.0193.

[22] Liu, G., Song, R., Guo, H., Perski, Z., Yue, H., Han, C., Fan, J. (2016), Filtering SAR interferometric phase noise using a split-window model, Remote Sensing Letters, 7(8), 800-809, doi: 10.1080/2150704X.2016.1187316.

[23] Lauknes, T.R., Zebker, H.A., Larsen, Y. (2011), InSAR deformation time series using an L1-norm small-baseline approach, IEEE Transactions on Geoscience and Remote Sensing, 49(1), 536-546, doi: 10.1109/TGRS.2010.2051951. 

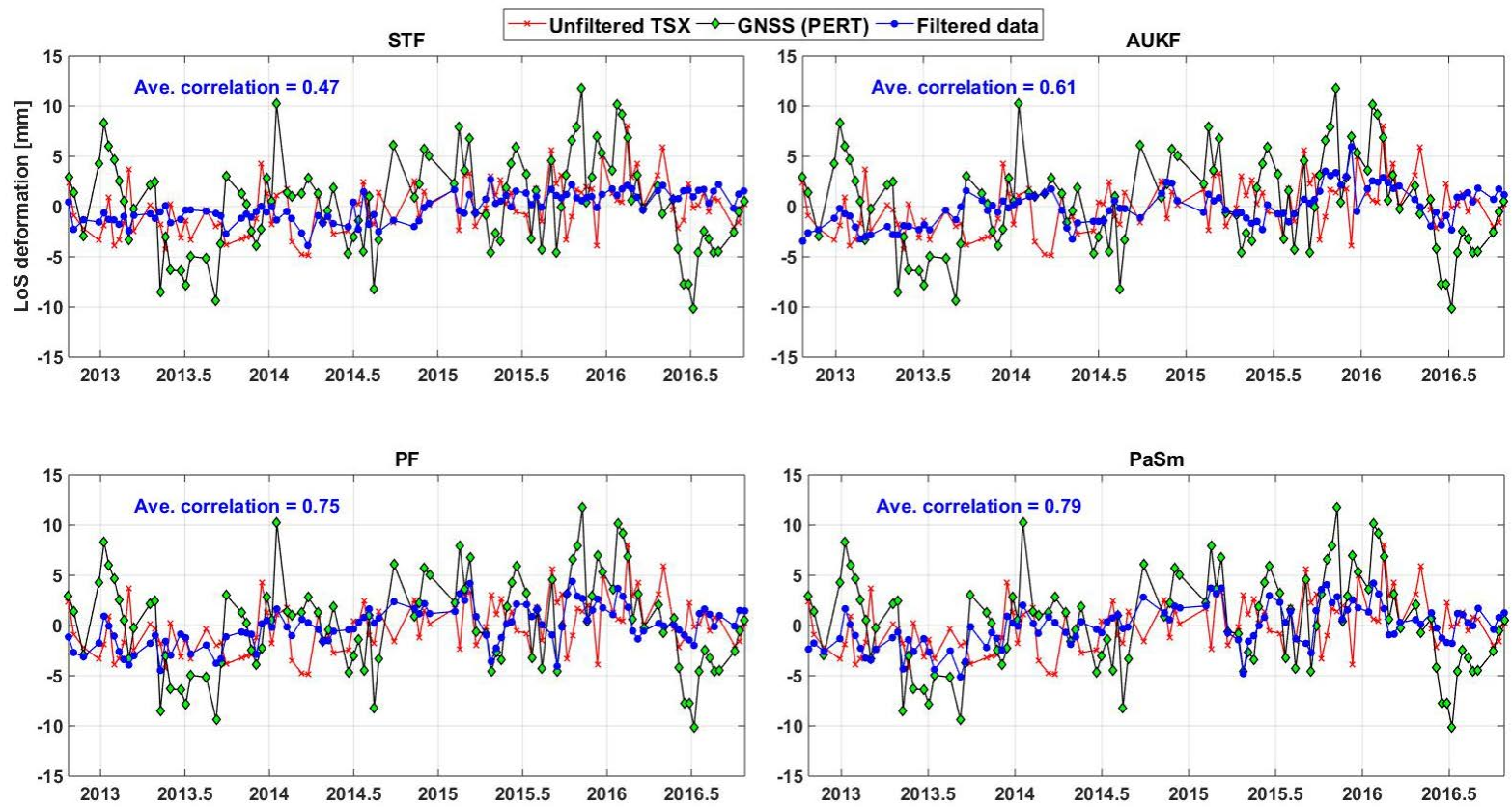

Fig. 5: InSAR time series at "PERT" station (latitude $-31.802^{\circ}$ and longitude $115.885^{\circ}$ ) from different filtering scenarios using TerraSar-X data compared to no filter and the GPS time series. Note that the correlation between the filtered times series and GPS is indicated on each subfigure. The correlation between noisy data and GPS time series is 0.18 .

[24] Biggs, J., Wright, T., Lu, Z., Parsons, B. (2007), Multi-interferogram method for measuring interseismic deformation: Denali Fault, Alaska, Geophysical Journal International, 170(3), 1165-1179, doi: 10.1111/j.1365-246X.2007.03415.x.

[25] Suo, Z., Li, Z., Bao, Z. (2010), A new strategy to estimate local fringe frequencies for InSAR phase noise reduction, IEEE Geoscience and Remote Sensing Letters, 7(4), 771-775, doi: 10.1109/LGRS.2010.2047935.

[26] Deledalle, C.-A., Denis, L., Tupin, F., Reigber, A., Jäger, M. (2015), NL-SAR: A unified nonlocal framework for resolution-preserving (Pol)(In)SAR denoising, IEEE Transactions on. Geoscience and. Remote Sensing, 53(4), 2021-2038, doi: 10.1109/TGRS.2014.2352555.

[27] Deledalle, C.A. (2009), Iterative weighted maximum likelihood denoising with probabilisic patch-based weights, IEEE Transactions on Image Processing, 18(12), 2661-2672, doi: 10.1109/TIP.2009.2029593.

[28] Takens, F. (1981), Lecture Notes in Math. Springer-Verlag: Berlin 898.

[29] Hamilton, F., Berry, T., Sauer, T. (2016), Ensemble Kalman filtering without a model, Physical Review X, 6(1), 011021, 6(1), doi: 10.1103/PhysRevX.6.011021.

[30] Khaki, M., Hamilton, F., Forootan, E., Hoteit, I., Awange, J., Kuhn, M. (2018), Non-parametric data integration scheme for land hydrological applications, Water Resources Research, 54(7), 4946-4964, doi: 10.1029/2018WR022854.

[31] Khaki, M., Hoteit, I., Kuhn, M., Forootan, E., Awange, J. (2019), Assessing data assimilation frameworks for using multi-mission satellite products in a hydrological context, Science of the Total Environment, 647, 1031-1043, doi: 10.1016/j.scitotenv.2018.08.032.

[32] Martinez-Espla, J.J., Martinez-Marin T., Lopez-Sanchez, J.M. (2009), A Particle Filter Approach for InSAR Phase Filtering and Unwrapping, in IEEE Transactions on Geoscience and Remote Sensing, 47(4), 1197 1211, doi: 10.1109/TGRS.2008.2008095.

[33] Gao, Y., Zhang, S., Li, T., Chen, Q., Li, S., Meng, P. (2018), Adaptive unscented Kalman filter phase unwrapping method and its application on Gaofen-3 interferometric SAR data, Sensors, 18(6), 1793, doi: $10.3390 / \mathrm{s} 18061793$.

[34] Koch, K.R., (2007), Introduction to Bayesian Statistics (2nd), Springer, Berlin.

[35] Khaki, M., Hoteit, I., Kuhn, M., Awange, J., Forootan, E., van Dijk, A.I.J.M., Schumacher, M., Pattiaratchi, C. (2017a), Assessing sequential data assimilation techniques for integrating GRACE data into a hydrological model, Advances in Water Resources, 107, 301-316, doi: 10.1016/j.advwatres.2017.07.001.
[36] Hamilton, F., Berry, T., Sauer, T. (2017), Kalman-Takens filtering in the presence of dynamical noise, The European Physical Journal Special Topics, 226:3239, doi: 10.1140/epjst/e2016-60363-2.

[37] Berry, T., Sauer, T. (2013), Adaptive ensemble Kalman filtering of nonlinear systems, Tellus A: Dynamic Meteorology and Oceanography, 65(1), 20331, doi: 10.3402/tellusa.v65i0.20331.

[38] Arulampalam, M.S., Maskell, S., Gordon, N., Clapp, T. (2002), A tutorial on particle filters for online nonlinear/non-Gaussian Baysian tracking, IEEE Transanctions on Signal Processings, 50(2), 174-188, doi: $10.1109 / 78.978374$.

[39] Doucet, A., Johanse, A.M. (2008), A tutorial on particle filtering and smoothing: fifteen years later. In: Crisan, D. and Rozovskii, B., Eds., The Oxford Handbook of Nonlinear Filtering, Oxford University Press, New York, 656-704.

[40] Wan, E., van der Merwe, R. (2001), The Unscented Kalman Filter, Wiley Publishing, ISBN: 0-471-36998-5.

[41] Klaas, M., Briers, M., de Freitas, N., Doucet, A., Maskell, S., Lang D. (2006), Fast Particle Smoothing: If I had a Million Particles, Machine Learning, Proceedings of the Twenty-Third International Conference (ICML 2006), Pittsburgh, Pennsylvania, USA, doi: 10.1145/1143844.1143905

[42] Doucet, A., Cappe, O., Moulines, E. (2005), Comparison of resampling schemes for particle filtering, ISPA 2005. Proceedings of the 4th International Symposium on Image and Signal Processing and Analysis, Zagreb, Croatia, 64-69, doi: 10.1109/ISPA.2005.195385.

[43] Breit, H., Fritz, T., Balss, U., Lachaise, M., Niedermeier, A., Vonavka, M. (2010), TerraSAR-X SAR Processing and Products, IEEE Transactions on Geoscience and Remote Sensing, 48(2), 727-740. doi: 10.1109/TGRS.2009.2035497.

[44] Kampes, B.M., Usai, S. (1999), Doris: The Delft Object-Oriented Radar Interferometric Software.

[45] Doucet, A., Freitas, N., Murphy, K., Russell, S. (2000), Rao blackwellised particle filtering for dynamic Bayesian networks, in C. Boutilier and M. Godszmidt (eds), Uncertainty in Artificial Intelligence, Morgan Kaufmann Publishers, San Francisco, CA, USA, 176-183.

[46] Fuhrmann, T., Garthwaite, M.C. (2019), Resolving Three-Dimensional Surface Motion with InSAR: Constraints from Multi-Geometry Data Fusion. Remote Sens. 2019, 11, 241. 